\title{
Validación de la Escala de Dificultades en la Regulación Emocional en la población universitaria de Córdoba, Argentina*
}

\author{
Validation of the Difficulties in Emotion Regulation Scale \\ in the University Population of Cordoba, Argentina
}

Recibido: junio 14 de 2013 | Revisado: marzo 2 de 2014 | Aceptado: abril 18 de 2014

\author{
LEONARDO AdRIÁN MEDRANO ** \\ Universidad Siglo 21, Córdoba, Argentina \\ MARIO TRÓGOLO *** \\ Universidad Siglo 21, Córdoba, Argentina
}

doi.org/10.11144/Javeriana.UPSY13-4.vedr

Para citar este artículo: Medrano, L. A., \& Trógolo, M. (2014). Validación de la Escala de Dificultades en la Regulación Emocional en la población universitaria de Córdoba, Argentina. Universitas Psychologica, 13(4), 1345-1356. http://dx.doi.org/10.11144/ Javeriana.UPSY13-4.vedr

* Artículo instrumental

** Universidad Siglo 21, Córdoba, Argentina. Dirección: León 1855. Córdoba, Argentina. Código Postal: 5000. Teléfono: 54-0351-4869998 (no fax). Correo electrónico: leomedpsico@gmail.com

**** Dirección: Avda. Colón 870, planta baja, departamento 4. Córdoba, Argentina. Teléfono: 54-03514241451 (no fax).

Correo electrónico: mario.trogolo@gmail.com

\section{RESUMEN}

En este estudio se presenta la adaptación de la Escala de Dificultades en la Regulación Emocional (DERS) para universitarios de Córdoba, Argentina. Mediante un muestreo accidental se seleccionaron 211 universitarios provenientes diferentes carreras. El análisis factorial exploratorio reveló la estructura original de seis factores subyacentes (50.79\% de la varianza total). Se realizaron estudios de consistencia interna y validez concurrente con las escalas de neuroticismo y extraversión del cuestionario IPIP-FFM evidenciando resultados satisfactorios. Finalmente, en estudios de contrastación según el género, solo el factor Falta de Claridad Emocional resultó superior en mujeres $(t=2.19 ; g l=209 ; p<0.05 ; d=0.17)$. Los resultados obtenidos son prometedores y posibilitan el desarrollo de nuevas investigaciones que indaguen el rol de la regulación emocional en universitarios cordobeses. Palabras clave

Escala de Dificultades en la Regulación Emocional; análisis psicométricos; universitarios

\section{A B S T R A C T}

The purpose of the present study was to validate the Difficulties in Emotion Regulation Scale (DERS) in the university population of Cordoba, Argentina. A convenience sample of 211 undergraduates enrolled in different careers was recruited. Exploratory factor analysis yielded a six-factor structure similar to the original version which explained $50.79 \%$ of the variance. Examination of internal consistency and concurrent validity with neuroticism and extraversion traits of IPIP-FFM revealed satisfactory results. Finally, studies examining gender differences showed a significant although small difference in the factor "lack of emotional clarity" for women $(t=2.19$; $d f=209$; $p<0.05 ; d=0.17)$. In general, results are promising and enable the development of further researches that examine the role of difficulties in emotion regulation at the university context of Cordoba.

Keywords difficulties in emotion regulation scale; psychometric properties; undergraduate 


\section{Introducción}

En la última década, el estudio de las emociones en el contexto académico ha sido objeto de gran interés tanto en la escuela media como en la universidad, observándose el impacto de las mismas sobre la motivación, el aprendizaje, el logro académico y la permanencia en los estudios (Pekrun, Goetz, Titz, $\&$ Perry, 2002). En este sentido, si bien el estudio de la ansiedad y sus efectos sobre el rendimiento es de larga data, recientemente se advierte en la literatura científica el desarrollo de investigaciones que analizan el papel de diferentes emociones frecuentemente experimentadas por los estudiantes sobre diversos aspectos del comportamiento académico (González, Donolo, \& Rinaudo, 2009; Pekrun, Molfenter, Titz, \& Perry, 2000; Turner \& Schallert, 2001).

Los estudiantes experimentan un amplio repertorio de emociones durante sus experiencias de aprendizaje en la universidad. Así, Pekrun et al. (2002) hallaron que los estudiantes comunican emociones de disfrute, esperanza, orgullo, alivio, enojo, ansiedad, desesperanza, vergüenza y aburrimiento frente a diferentes situaciones académicas. En otro estudio similar (Medrano \& Vilte, 2010) se observó que los estudiantes universitarios tienden a experimentar emociones positivas (p. ej., orgullo, interés, disfrute) fundamentalmente en situaciones de éxito académico, apoyo social, comprensión de contenidos interesantes y asistencia a clases dinámicas y participativas. Por otra parte, se observó una amplia variedad de situaciones que pueden provocar emociones negativas (p. ej., aburrimiento, vergüenza, ansiedad), tales como el pobre desempeño en exámenes, la asistencia a clases netamente expositivas, el estudio de contenidos aburridos o poco comprensibles, la desorganización institucional y las huelgas docentes, entre otras.

En general, las investigaciones realizadas indican que las emociones positivas poseen una relación directa y significativa con la motivación, el aprendizaje y el rendimiento académico, mientras que las emociones negativas presentan una relación inversa y significativa con dichas variables (González et al., 2009; Medrano \& Moretti, 2013). Sin embargo, algunas evidencias sugieren que dichos patrones no son consistentes. En efecto, se ha encontrado que algunas emociones negativas como la ansiedad, la vergüenza y el enojo, debido a su naturaleza activadora, pueden tener un efecto reforzador sobre la motivación y el rendimiento (Bandura \& Cervone, 1983; Pekrun \& Hofmann, 1999; Turner \& Schallert, 2001). Del mismo modo, ciertas emociones positivas desactivadoras, como el alivio o la relajación, pueden disminuir la motivación y el engagement sobre la tarea inmediata, influyendo negativamente sobre el rendimiento (Pekrun et al., 2002). De esta manera, la relación entre las emociones y el comportamiento académico es compleja, pudiendo facilitar u obstaculizar el comportamiento académico óptimo de los estudiantes.

Tomando en consideración los antecedentes señalados, un factor relevante en el comportamiento de los estudiantes parece estar asociado con la capacidad para regular de manera efectiva las emociones. En términos amplios, la regulación emocional comprende los procesos mediante los cuales las personas influyen sobre las emociones que tienen, cuándo las tienen y el modo en que expresan y experimentan las mismas (Gross, 1998). En este sentido, existe considerable acuerdo en torno a la idea de que las emociones no son fuerzas incoercibles, sino que las personas son capaces de incrementar, mantener, o disminuir sus emociones positivas y negativas cuando lo deseen o necesitan; por lo tanto, son capaces de modificar su conducta dependiendo de sus intereses, necesidades o de las situaciones en las que se encuentran (Koole, 2009).

Investigaciones llevadas a cabo en el ámbito académico han evidenciado que la capacidad para regular las emociones constituye una variable fuertemente asociada al rendimiento académico (Graziano, Reavis, Keane, \& Calkins, 2007; Gumora \& Arsenio, 2002) la satisfacción académica (Trógolo \& Medrano, 2012) y la calidad de las relaciones sociales entre pares (Lopes, Salovey, Cotê, \& Beers, 2005), mientras que la dificultad para regular las emociones se ha relacionado con distintos comportamientos disfuncionales tales como comportamientos agresivos y antisociales hacia los compañeros y docentes (Graziano et al., 
2007) y con problemas de aprendizaje (Eisenberg et al., 1996).

Aunque estos estudios indican que la regulación emocional de los estudiantes constituye un factor de importancia para favorecer el comportamiento académico óptimo, en nuestro contexto no se han realizado estudios que brinden evidencia empírica al respecto debido en parte a la ausencia de instrumentos que posibiliten una medición válida y confiable. Contar con un instrumento de tales características sería de gran utilidad para desarrollar estudios tendientes a evaluar la relación entre la regulación emocional y otros comportamientos académicos de importancia, identificar estudiantes con déficits en la regulación de las emociones y, finalmente, evaluar el impacto de intervenciones dirigidas a incrementar las capacidades para regular las emociones de los estudiantes. Atendiendo a esto, el presente trabajo se desarrolló con el objetivo de validar la Escala de Dificultades en la Regulación Emocional ([DERS]; Gratz \& Roemer, 2004) en la población de universitarios de Córdoba.

Este instrumento fue originalmente desarrollado por Gratz y Roemer (2004) con el propósito de identificar dificultades clínicamente relevantes de regulación de emociones negativas. Para ello, los autores efectuaron una revisión de diferentes modelos conceptuales sobre regulación de emociones y propusieron uno propio, sobre la base del cual elaboraron el DERS.

Tradicionalmente, la regulación emocional ha sido conceptualizada sobre la base del control sobre la experiencia y expresión emocional. En contraposición, otros modelos enfatizan que la regulación no es sinónimo de control emocional, dado que debe atenderse a la funcionalidad de la emoción y no solo a la disminución de un estado emocional displacentero. Incluso la incapacidad para aceptar estados emocionales negativos puede fortalecer dichas emociones en vez de atenuarlas (Stewart, Zvolensky, \& Eifert, 2002). En este sentido, se plantea que una regulación adaptativa de las emociones implica un monitoreo y evaluación de la experiencia emocional además de un posible control sobre esta, destacando de esta forma la importancia del entendimiento y la conciencia sobre los estados emocionales.
Sobre la base de esta conceptualización Gratz y Roemer (2004) afirman que una regulación adaptativa de las emociones consiste en modular las experiencias emocionales en vez de eliminarlas. Esta modulación de la activación emocional estaría al servicio de reducir la urgencia asociada a la emoción y aumentar el control que la persona tiene sobre su comportamiento. Así, la regulación emocional referiría a la capacidad para inhibir impulsos o conductas inapropiadas o desadaptativas. De esta manera, personas con mayores capacidades de regulación emocional lograrían poner en marcha los comportamientos necesarios para lograr las metas planteadas o responder satisfactoriamente a las demandas de la situación, aún bajo los efectos de un estado emocional displacentero. Sobre la base de este modelo, proponen que la regulación emocional involucraría cuatro dimensiones: a) conciencia y claridad emocional, b) aceptación de emoción, c) habilidad para mantener el comportamiento dirigido a una meta y el control de impulsos y d) acceso a estrategias de regulación emocional. De esta manera, las dificultades en regulación emocional provendrían de la ausencia relativa de alguna o todas las dimensiones mencionadas. No obstante, a partir de los resultados obtenidos mediante análisis factorial los autores desglosaron la dimensión de conciencia y claridad en dos dimensiones, y diferenciaron el control de impulsos y la interferencia en metas en dos dimensiones, quedando así un total de seis dimensiones.

Cabe señalar que este modelo difiere al utilizado por otros instrumentos, como por ejemplo la Escala de Regulación del Humor Negativo (Negative Mood Regulation Scale [NMR]; Catanzaro \& Mearns, 1990) que evalúa comportamientos y cogniciones tendientes a aliviar una emoción negativa e inducir emociones positivas, lo cual enfatiza el modelo de eliminación y evitación de emociones negativas que critican Gratz y Roemer (2004). Algo semejante se observa en relación con la Escala de Metaconocimientos sobre Estados Emocionales (Trait Meta-Mood Scale [TMMS]; Salovey, Mayer, Goldman, Turvey, \& Palfai, 1995) basada en el modelo de inteligencia emocional. Si bien este instrumento evalúa aspectos no contemplados 
por el NMR, enfatiza la evitación de emociones displacenteras y no incluye reactivos que evalúen la capacidad de mantener un comportamiento en la dirección deseable bajo los efectos de una emoción negativa. Por otra parte, cabe mencionar que la TMMS ya cuenta con una versión validada para la población Argentina (Calero, 2013). Otro instrumento ampliamente utilizado para evaluar regulación emocional es la Escala de Regulación Emocional Cognitiva (Cognitive Emotion Regulation Questionnaire [CERQ]; Garnefski, Kraaij, \& Spinhoven, 2001). No obstante, la escala CERQ se limita a examinar los procesos cognitivos que las personas utilizan luego de experimentar un evento negativo, por lo que refiere exclusivamente a estrategias cognitivas de regulación y no contemplan estrategias conductuales. Este instrumento también cuenta con una validación para la población Argentina con propiedades psicométricas aceptables (Medrano, Moretti, Ortiz, \& Pereno, 2013).

ElDERS ha sido adaptado a diferentes culturas, aunque con resultados psicométricos dispares. De esta manera, el instrumento cuenta con versiones italiana (Sighinolfi, Norcini, \& Rocco, 2010), turca (Ruganci \& Genöz, 2010), portuguesa (Coutinho, Ribeiro, Ferreirinha, \& Dias, 2009) y alemana (Newman, van Lier, Gratz, \& Koot, 2010) con propiedades psicométricas aceptables y semejantes a la versión original. Solo se encontraron dos adaptaciones del DERS para culturas de lengua castellana: España (Hervás \& Jódar, 2008) y México (Tejeda, García, González-Forteza, \& Palos, 2012); en ambos casos existieron variaciones atendibles en la estructura factorial del DERS. En la versión de Hervás y Jódar, se observó la existencia de un factor menos, ya que el análisis factorial efectuado no discriminaba entre los ítems correspondientes a las dimensiones Dificultades en el Control de Impulsos y Acceso Limitado a Estrategias de Regulación, por lo cual los autores optaron por agrupar dichas dimensiones en un mismo factor denominado Descontrol Emocional. Los estudios psicométricos reportaron valores adecuados de consistencia interna (valores alfa de Cronbach comprendidos entre 0.73 y 0.91 para las distintas escalas), de fiabilidad test-retest, y evidencias aceptables de validez convergente e incremental. Por su parte, en la versión mexicana de Tejeda y colaboradores, los estudios de análisis factorial exploratorio y confirmatorio indicarían que un modelo de cuatro factores presenta un mejor ajuste a los datos. En esta versión, se eliminaron 12 reactivos y los 24 ítems restantes se agruparon en las dimensiones: no aceptación de las respuestas emocionales, dificultades para dirigir el comportamiento hacia metas, falta de conciencia emocional y falta de claridad emocional. Más allá de esta diferencia en la estructura factorial, se obtuvieron resultados satisfactorios en los estudios de consistencia interna y validez externa.

Aunque el DERS fue desarrollado inicialmente con el objetivo de ser utilizado en el campo de la psicología clínica, estudios posteriores destacan su utilidad en muestras no clínicas como por ejemplo universitarios (Hervas \& Jódar, 2008; Tejeda et al., 2012). Tal como señalan Gratz y Roemer (2004), no es posible evaluar y comprender la regulación emocional sin considerar el contexto específico en el que se desarrolla, las demandas situacionales y las metas individuales. Sin una información precisa del contexto no se puede determinar si se está haciendo un uso funcional y adaptativo de las estrategias de regulación emocional. Atendiendo a ello, en el presente estudio instrumental, se examinaron las propiedades psicométricas del DERS en la población Argentina de universitarios.

\section{Método}

\section{Participantes}

Participaron un total de 211 estudiantes universitarios provenientes estudiantes de diferentes carreras (Psicología, Medicina, Abogacía, Arquitectura, de Contador Público e Ingeniería) de universidades públicas $(74.1 \%)$ y privadas $(25.9 \%)$ de la ciudad de Córdoba, Argentina. Se incluyeron en la muestra estudiantes de ambos sexos (mujeres $=65.4 \%$; hombres $=33.6 \%$ ), con edades comprendidas entre los 18 y 53 años $(M=22.25 ; D E=3.64)$. Los participantes fueron seleccionados mediante un muestro no probabilístico de tipo accidental. Cabe destacar que el tamaño muestral cumple con los 
criterios estipulado por Hogan (2004), de contar al menos con 5 participantes por ítem.

\section{Instrumentos}

Escala de Dificultades en la Regulación

Emocional ([DERS]; Gratz Eु Roemer, 2004)

Se utilizó la escala inicialmente desarrollada por Gratz y Roemer (2004) y luego adaptada a la población española por Hervás y Jódar (2008). La escala original cuenta con 36 ítems con un formato de respuesta Likert de 5 posiciones (desde $1=$ casi nunca hasta $5=$ casi siempre) y una estructura de seis factores: 1) Dificultades en el Control de Impulsos (6 ítems: "Cuando me enfado, pierdo el control sobre mis comportamientos", por ejemplo); 2) Acceso Limitado a Estrategias de Regulación Emocional (8 ítems: "Cuando me disgusto, creo que no hay nada que pueda hacer para sentirme mejor", por ejemplo); 3) Falta de Aceptación Emocional (6 ítems: "Cuando me disgusto, me siento culpable por sentirme así”, por ejemplo); 4) Interferencia en Conductas Dirigidas a Metas ( 5 ítems: "Cuando me altero, me cuesta concentrarme”, por ejemplo); 5) Falta de Conciencia Emocional (6 ítems invertidos: "Estoy atento/a a mis sentimientos", por ejemplo) y 6) Falta de Claridad Emocional (5 ítems: "me siento confuso/a acerca de cómo me siento", por ejemplo). Cabe señalar que las diferentes escalas presentan una elevada consistencia interna (valores $\alpha$ comprendidos entre 0.8 y 0.89 ) así como también la escala total $(\alpha=0.93)$.

Cuestionario de personalidad IPIP-FFM (Gross, Salazar-Jaime, Piccolo, $\mathcal{E}$ Cupani, 2012)

Este cuestionario se compone de 50 ítems redactados en forma de frase, construido sobre la base del modelo de los Cinco Grandes Factores (FFM, por sus siglas en inglés) de personalidad. Cada frase describe comportamientos típicos de las personas vinculados a los diferentes rasgos de personalidad propuestos por el modelo: extraversión (10 ítems: "Generalmente comienzo las conversaciones", por ejemplo), neuroticismo (10 ítems: "Tengo cambios frecuentes en el estado de ánimo”, por ejemplo), responsabilidad (10 ítems: "Realizo mis tareas inmediatamente", por ejemplo), amabilidad (10 ítems: "Dedico tiempo a otras personas", por ejemplo) y apertura la experiencia o intelecto (10 ítems: “Tengo una imaginación fluida", por ejemplo). Para completar la prueba, se le solicita al sujeto que evalúe el grado de precisión con que cada oración lo describe, utilizando una escala tipo Likert de cinco opciones de respuesta (desde $1=$ Muy en desacuerdo con esta descripción de mí mismo; hasta $5=$ Muy de acuerdo con esta descripción de mí mismo). Los estudios psicométricos revelaron valores satisfactorios de consistencia interna (valores $\alpha$ comprendidos entre 0.75 y 0.86 ) y aportaron evidencias de validez predictiva de la escala.

\section{Procedimiento y análisis de datos}

Se realizó un estudio de tipo instrumental (Montero \& León, 2007), siguiendo los criterios establecidos por la normativa psicométrica internacional (American Psychological Association [APA], 1999). La administración de los instrumentos se realizó por tres colaboradores debidamente entrenados. La participación fue voluntaria y todos los participantes fueron informados acerca de los fines de la investigación y del carácter anónimo y confidencial de sus respuestas, antes de obtener su consentimiento. Los datos obtenidos fueron cargados y analizados en el paquete estadístico SPSS 17.0 y en el programa Vista en su versión 6.

\section{Resultados}

\section{Preparación de los datos}

Siguiendo las recomendaciones de la literatura especializada (Uriel \& Aldas, 2005), previo al análisis, se examinó la presencia de valores perdidos y la existencia de casos atípicos. Solo el ítem 3 ("Yo experimento mis emociones como sobrecogedoras y fuera de control") presentó un porcentaje superior al 5\% de casos perdidos. Al examinar el patrón de los casos perdidos para este ítem, se observó que dicho patrón no era aleatorio al considerar las 
respuestas para los ítems 21 ("Cuando me altero, me siento avergonzado/a por sentirme así”) y 23 ("Cuando me altero, siento que soy débil"). En efecto, los participantes que no respondían al ítem 3 presentaban puntajes significativamente menores en los ítems $21(t=4.1 ; g l=21.5 ; p<0.001)$ y 23 $(t=4.2 ; g l=17.9 ; p<0.001)$. Tomando esto en consideración, se tendrán ciertas precauciones al momento de interpretar el comportamiento de los ítems mencionados.

Por otra parte, se obtuvieron puntajes $z$ de cada ítem, observándose 31 casos atípicos univariados (valores comprendidos fuera del rango $z \pm 3$; Tabachnick \& Fidell, 2001). Asimismo, se identificaron 11 casos atípicos multivariados mediante el procedimiento estadístico de distancia de Mahalanobis $\left(D^{2}\right)$. Considerando que los casos atípicos pueden distorsionar las relaciones entre los ítems y en consecuencia el análisis factorial, se optó por realizar el análisis factorial con y sin casos atípicos para examinar la convergencia de las soluciones factoriales, calculando un coeficiente de correlación de Pearson a partir de las matrices de saturaciones obtenidas luego de la rotación (Rivas, 1999). Los resultados sugieren una elevada congruencia o similaridad entre los factores (valores $r$ comprendidos entre 0.93 y 0.98), por lo cual se mantuvieron los casos atípicos en la muestra.

Se obtuvieron los índices de asimetría y curtosis para cada uno de los ítems. Todos los ítems presentaron valores comprendidos entre \pm 2 sugiriendo una distribución normal de acuerdo con los criterios propuestos por George y Mallery (2010), excepto el ítem 4 ("No tengo ni idea de cómo me siento"). Teniendo en cuenta esto y que estudios posteriores señalaron que el mismo provocaba una disminución considerable en el coeficiente alfa de Cronbach (el valor $\alpha$ decrecía de 0.7 a 0.45 ), se optó por eliminar este ítem.

\section{Análisis de estructura interna}

Se realizó un análisis factorial exploratorio con los 35 ítems para determinar la estructura interna del DERS, utilizando como método de extracción Mínimos Cuadrados no Ponderados. Se optó por el uso de este método debido a que los datos presentaron un notable apartamiento de la distribución normal multivariada (índice de Mardia = 205.689), de acuerdo con los criterios reportados por Rodríguez Ayán y Ruiz (2008), siendo recomendable en estos casos como así también cuando el tamaño muestral no es elevado, tal como ocurre en la presente investigación (Ximénez \& García, 2005). La medida de adecuación muestral Kaiser-Meyer-Olkin (KMO $=0.85)$ y la prueba de esfericidad de Bartlett $\left(\chi^{2}\right.$ $=2819,03 ; g l=595 ; p<0.001)$ sugirieron una intercorrelación adecuada entre los ítems para la aplicación del análisis factorial.

La regla Kaiser-Gutman de autovalores superiores a 1 sugirió la existencia de 8 factores que explican en conjunto el $62.64 \%$ de la variabilidad de la prueba. Sin embargo, dado que este método tiende a sobreestimar la cantidad de factores subyacentes (Thompson, 2004), se estimó la cantidad de factores mediante el gráfico de sedimentación (scree plot) y el análisis factorial paralelo (Ledesma \& Valero-Mora, 2007). Ambos métodos sugirieron una estructura de seis factores, por lo cual se optó por analizar los ítems solicitando la extracción de seis factores. Tal como sugieren Costello y Osborne (2005), para interpretar la solución factorial obtenida se llevó a cabo una rotación oblicua PROMAX ya que algunos de los factores presentaban correlaciones superiores a 0.3 (Tabla 1).

Considerando que 7 ítems presentaron cargas factoriales superiores a 0.3 en más de un factor (ítems 10, 15, 16, 17, 23, 24 y 31), se optó por eliminarlos a los fines de trabajar con ítems unidimensionales. De este modo, la escala quedó conformada por 28 ítems agrupados en 6 factores que explican el $50.79 \%$ de la varianza total.

\section{Análisis de consistencia interna}

Para examinar la consistencia interna de cada escala se utilizó el coeficiente alfa de Cronbach, obteniendo los siguientes valores: falta de conciencia emocional $\alpha=0.71$, falta de claridad emocional $\alpha=0.7$, falta de aceptación emocional $\alpha=0.84$, interferencia en conductas dirigidas a metas $\alpha=$ 0.82 , dificultades en el control de los impulsos $\alpha$ 
TABLA 1

Estructura factorial del DERS (matriz rotada)

\begin{tabular}{|c|c|c|c|c|c|c|}
\hline & \multicolumn{6}{|c|}{ Factores } \\
\hline & $\begin{array}{c}\text { Falta de } \\
\text { aceptación } \\
\text { emocional }\end{array}$ & $\begin{array}{c}\text { Interferencia en } \\
\text { conducta dirigida } \\
\text { a metas }\end{array}$ & $\begin{array}{l}\text { Dificultades en } \\
\text { el control de los } \\
\text { impulsos }\end{array}$ & $\begin{array}{c}\text { Falta de } \\
\text { conciencia } \\
\text { emocional }\end{array}$ & $\begin{array}{c}\text { Falta de claridad } \\
\text { emocional }\end{array}$ & $\begin{array}{c}\text { Acceso limitado } \\
\text { a estrategias de } \\
\text { regulación }\end{array}$ \\
\hline DERS 12 & 0.84 & & & & & \\
\hline DERS 21 & 0.82 & & & & & \\
\hline DERS 29 & 0.74 & & & & & \\
\hline DERS 25 & 0.67 & & & & & \\
\hline DERS 30 & 0.63 & & & & & \\
\hline DERS 11 & 0.39 & & & & & \\
\hline DERS 26 & & 0.84 & & & & \\
\hline DERS 18 & & 0.77 & & & & \\
\hline DERS 33 & & 0.75 & & & & \\
\hline DERS 20 & & 0.58 (i) & & & & \\
\hline DERS 13 & & 0.62 & & & & \\
\hline DERS 19 & & & 0.94 & & & \\
\hline DERS 14 & & & 0.92 & & & \\
\hline DERS 32 & & & 0.76 & & & \\
\hline DERS 27 & & & 0.54 & & & \\
\hline DERS 36 & & & 0.44 & & & \\
\hline DERS 3 & & & 0.36 & & & \\
\hline DERS 6 & & & & $0.7(\mathrm{i})$ & & \\
\hline DERS 8 & & & & $0.67(\mathrm{i})$ & & \\
\hline DERS 2 & & & & 0.66 (i) & & \\
\hline DERS 34 & & & & $0.45(\mathrm{i})$ & & \\
\hline DERS 9 & & & & & 0.65 & \\
\hline DERS 5 & & & & & 0.57 & \\
\hline DERS 7 & & & & & 0.58 (i) & \\
\hline DERS 1 & & & & & 0.44 (i) & \\
\hline DERS 22 & & & & & & 0.68 (i) \\
\hline DERS 35 & & & & & & 0.41 \\
\hline DERS 28 & & & & & & 0.45 \\
\hline
\end{tabular}

Nota. (i) = Ítems con puntuación invertida.

Fuente: elaboración propia

$=0.87$, acceso limitado a estrategias de regulación emocional $\alpha=0.54$.

\section{Validez concurrente}

Diferentes estudios han demostrado que un factor importante relacionado con la capacidad para regular las emociones son los rasgos de personalidad, fundamentalmente los rasgos de neuroticismo y extraversión. En este sentido, la evidencia previa indica que altos niveles de neuroticismo se aso- cian con el uso de estrategias menos efectivas de regulación emocional, mientras que la extraversión ha sido relacionada con el uso de estrategias más efectivas de regulación (John \& Gross, 2004; Niven, Totterdell, Stride, \& Holman, 2011; Wang, Shi, \& $\mathrm{Li}, 2009)$. Teniendo en cuenta esto, se correlacionaron las diferentes escalas del DERS con los rasgos de neuroticismo y extraversión (Tabla 2).

Tal como se esperaba, se hallaron relaciones positivas y significativas entre las diferentes escalas del DERS y el rasgo neuroticismo (valores $r$ 
comprendidos entre -0.15 y -0.5). Asimismo, varios factores del DERS correlacionaron de manera negativa y significativa con extraversión (valores $r$ entre -0.14 y -0.31 ).

\section{Diferencias en función del sexo o la edad}

Finalmente, se procedió evaluar la existencia de diferencias en las puntuaciones del DERS en función del género y la edad de los participantes. Luego de verificar el supuesto de homocedasticidad mediante el test de Levene, se aplicaron sucesivas pruebas $t$ para muestras independientes para determinar si existían diferencias estadísticamente significativas entre hombres $(n=72)$ y mujeres $(n=139)$. Dado que la probabilidad de cometer un error tipo 1 aumenta al calcular múltiples pruebas $t$ (Pagano, 1998), se llevó a cabo una corrección de Bonferroni para mantener los valores críticos de $t$ en un nivel $\alpha=0.05$. Solo se observaron diferencias estadísticamente significativas en la subescala Falta de Claridad Emocional $(t=2.19 ; g l=209 ; p<0.05$ con corrección Bonferroni). Aparentemente, las mujeres $(M=9.76)$ presentan mayores dificultades para diferenciar sentimientos que los hombres $(M$ $=8.83)$, sin embargo el tamaño del efecto de la diferencia es bajo $(d=0.17)$.

Para evaluar si existía relación entre las puntuaciones del DERS y la edad de los participantes se correlacionaron ambas variables mediante el coeficiente Rho de Spearman. Se optó por utilizar una prueba no paramétrica, ya que la variable edad pre- sentaba una marcada asimetría. No se observaron relaciones estadísticamente significativas (valores $r$ comprendidos entre -0.11 y 0.17 ).

\section{Discusión}

El objetivo del presente trabajo consistió en evaluar la fiabilidad y validez de la Escala de Dificultades en la Regulación Emocional ([DERS]; Gratz \& Roemer, 2004) en la población de estudiantes universitarios de Córdoba, Argentina. Los resultados obtenidos proveen evidencia preliminar sobre el funcionamiento psicométrico del DERS en el contexto universitario de Argentina. Tal como se sugiere en el estudio original (Gratz \& Roemer, 2004) y se observa en la mayoría de las adaptaciones posteriores (Coutinho et al., 2009; Newman et al., 2010; Ruganci \& Genöz, 2010; Sighinolfi et al., 2010), el DERS presenta una estructura factorial compuesta de seis dimensiones:

1) Falta de Aceptación Emocional: integrada por ítems que reflejan la tendencia a experimentar emociones negativas secundarias como respuesta a una emoción negativa primaria (ítem de mayor carga factorial: "Cuando me disgusto, me da vergüenza sentirme asî”).

2) Interferencia en Conductas Dirigidas a Metas: compuesto por ítems que refieren a dificultades para concentrarse o realizar tareas cuando se experimenta una emoción negativa (ítem de mayor carga factorial: "Cuando me altero, me cuesta concentrarme").

TABLA 2

Correlaciones entre los diferentes factores del DERS y los rasgos de neuroticismo y extraversión

\begin{tabular}{|c|c|c|c|c|c|c|c|c|}
\hline Factores & 1 & 2 & 3 & 4 & 5 & 6 & 7 & 8 \\
\hline 1 Falta de aceptación emocional & - & $0.39 * *$ & $-0.54 * *$ & 0.01 & $0.31 * *$ & $0.28 * *$ & $0.43 *$ & $-0.14 *$ \\
\hline 2 Interferencia en conductas dirigidas a metas & & - & $-0.51 * *$ & 0.11 & $0.35 * *$ & $0.35 * *$ & $0.39 * *$ & -0.09 \\
\hline 3 Dificultades en el control de impulsos & & & - & 0 & $-0.37 * *$ & $-0.31 * *$ & $0.4^{* *}$ & -0.12 \\
\hline 4 Falta de conciencia emocional & & & & - & $0.35 * *$ & $0.16^{*}$ & $0.15^{*}$ & $-0.31 * *$ \\
\hline 5 Falta de claridad emocional & & & & & - & $0.19 * *$ & $0.49 * *$ & $-0.18 * *$ \\
\hline 6 Acceso limitado a estrategias de regulación emocional. & & & & & & - & $0.5^{* *}$ & $-0.24 * *$ \\
\hline 7 Neuroticismo & & & & & & & - & $-0.36 * *$ \\
\hline 8 Extraversión & & & & & & & & - \\
\hline
\end{tabular}

$* p<0.05 . * * p<0.01$ (bilateral).

Fuente: elaboración propia 
3) Dificultades en el Control de Impulsos: este factor subyace a ítems que expresan dificultades para controlar el propio comportamiento cuando se experimenta una emoción negativa (ítem de mayor carga factorial: "Cuando me altero, me siento fuera de control").

4) Falta de Conciencia Emocional: factor conformado por reactivos que reflejan la tendencia a atender y admitir estados emocionales (ítem de mayor carga factorial: "Estoy atento/a a mis sentimientos"). Los ítems deben invertirse para evaluar la Falta de Conciencia Emocional, tal como se plantea en la escala original.

5) Falta de Claridad Emocional: integrado por ítems que examinan la medida en que una persona conoce y comprende sus emociones (ítem de mayor carga factorial: "Me siento confuso/a acerca de cómo me siento").

6) Acceso Limitado a Estrategias de Regulación Emocional: este último factor se encuentra compuesto por ítems que indican la creencia de que no se podrán modificar un estado emocional displacentero (ítem de mayor carga factorial (saturación negativa): "Cuando me disgusto, sé que puedo encontrar una forma de sentirme mejor eventualmente").

Es conveniente señalar que en el proceso de validación se eliminó un total de 8 reactivos que presentó pesos factoriales significativos en más de un factor, permaneciendo así 28 de los 36 ítems originales. El hecho de que estos ítems no hayan cargado en una única dimensión del DERS puede atribuirse a múltiples factores. En primer lugar debe considerarse que existen relaciones fuertes y moderadas entre las dimensiones del DERS, por lo cual resulta dificultoso que todos los ítems de la escala sean unidimensionales. Esta dificultad se observa sobre todo en las adaptaciones en idioma español que se han realizado del DERS (Hervás \& Jódar, 2008; Tejeda et al., 2012), donde se aprecia un disminución en la cantidad de factores debido al solapamiento de los reactivos. Por otra parte, factores socioculturales e idiomáticos pueden haber afectado el funcionamiento de los ítems. Tal es el caso de la expresión "revolcarme en mi disgusto" del ítem 23, la cual es poco utilizada en nuestra cultura. A pesar de estas variaciones y de contar con menos ítems que la versión original, cabe destacar que se mantuvo la estructura factorial original y se explicó una proporción semejante de la varianza total de la prueba.

En lo referido a la consistencia interna se obtuvieron valores óptimos y aceptables para las diferentes escalas del DERS. Sin embargo, los valores observados fueron en general inferiores a los reportados en estudios previos (Hervás \& Jódar, 2008; Gratz \& Roemer, 2004; Ruganci \& Gencöz, 2010), especialmente en el factor Acceso Limitado a Estrategias de Regulación Emocional. Una posible razón de la baja confiabilidad que presentó esta escala podría deberse a la reducción significativa en el número de ítems. Por consiguiente, sería provechoso efectuar estudios agregando nuevos ítems a dicho factor.

Por otra parte, el examen de la matriz de correlaciones entre las diferentes escalas del DERS y los rasgos de neuroticismo y extraversión mostró relaciones significativas en la dirección esperada. Concretamente, se observa que universitarios con mayores dificultades para regular sus emociones presentan mayores niveles de neuroticismo y que estudiantes que realizan un uso más adaptativo de sus estrategias de regulación emocional reportan mayores niveles de extraversión tal como se plantea en estudios antecedentes (John \& Gross, 2004; Niven et al., 2011; Wang et al., 2009). Se obtuvieron así evidencias satisfactorias de validez externa.

En cuanto a los estudios de contrastación grupos, en general no se observaron diferencias significativas en los puntajes del DERS entre hombres y mujeres, de manera similar a los resultados obtenidos en trabajos previos (Gratz \& Roemer, 2004; Hervás \& Jódar, 2008; Ruganci \& Gencöz, 2010). Solo se apreciaron diferencias significativas en el factor Falta de Claridad Emocional en favor de las mujeres; no obstante, estas diferencias fueron leves considerando los criterios reportados por Cohen (1988). Tampoco se observó que la edad se relacionara con las puntuaciones del DERS. Sin embargo, debe considerarse que la muestra no presentaba una alta variabilidad en cuanto a la edad. Esta restricción del rango (Aron \& Aron, 2001) pudo haber afectado el grado de la correlación. 
Entre las limitaciones del presente estudio, cabe considerar que la muestra estuvo conformada en mayor proporción por mujeres, y que sería deseable contar con un tamaño muestral superior. Por otra parte, se requiere de más estudios psicométricos tendientes a examinar la estabilidad de la escala y su poder predictivo. Asimismo, resultaría conveniente realizar estudios de análisis factorial confirmatorio, para determinar con mayor seguridad la cantidad de dimensiones subyacentes. En efecto, si bien la estructura factorial obtenida es coherente con la estructura obtenida en la escala original (Gratz \& Roemer, 2004) y con la mayor parte de las versiones adaptadas a otras culturas, una cantidad atendible de trabajos señalan una existencia menor de dimensiones (Hervás \& Jódar, 2008; Ruganci \& Gencöz, 2010; Tejeda et al., 2012).

Más allá de las limitaciones señaladas, los resultados obtenidos en el presente estudio son prometedores y respaldan la utilización del DERS en la población universitaria de Córdoba, Argentina. Dicho estudio resulta de gran valor, ya que permite disponer de un instrumento útil para el desarrollo de investigaciones en nuestro medio que indaguen el rol de las dificultades en la regulación emocional en el ámbito universitario. Concretamente, se prevé realizar diferentes estudios tendientes a analizar la relación entre la dificultad para regular las emociones y diferentes constructos académicos de importancia como la autoeficacia, el estrés, el aprendizaje autorregulado, el rendimiento académico y el progreso en las metas académicas. Por otro lado, se pretende, mediante la utilización del DERS, identificar estudiantes universitarios con baja capacidad para regular sus emociones y evaluar el impacto de intervenciones dirigidas a incrementar dicha capacidad. En suma, el presente trabajo abre un potencial abanico de investigaciones e intervenciones prácticas, orientadas a estudiar y promover la regulación emocional en el medio universitario de Córdoba.

\section{Referencias}

American Psychological Association. (1999). Standards for psychological and educational tests. Washington, DC: Autor.
Aron, A., y Aron. E. (2001). Estadística para Psicología. Argentina: Pearson Education

Bandura, A., \& Cervone, D. (1983). Self-evaluative and self-efficacy mechanisms governing the motivational effects of goal systems. Journal of Personality and Social Psychology, 45(5), 1017-1028.

Calero, A. (2013). Versión argentina del TMMS para adolescentes: una medida de la inteligencia emocional percibida. Cuadernos de Neuropsicología, 7(1), 104-119.

Catanzaro, S., \& Mearns, J. (1990). Measuring generalized expectancies for negative mood regulation: Initial scale development and implications. Journal of Personality Assessment, 54(3-4), 546-563.

Cohen, J. (1988). Statistical power analysis for the behavioral science (2.a ed.). Hillsdale, NJ: Erlbaum.

Costello, A., \& Osborne, J. (2005). Best practices in exploratory factor analysis: Four recommendations for getting the most from your analysis. Practical Assessment, Research EF Evaluation, 10(7), 1-9.

Coutinho, J., Ribeiro, E., Ferreirinha, R., \& Dias, P. (2009). The Portuguese version of the Difficulties in Emotion Regulation Scale and its relationship with psychopathological symptoms. Revista de Psicología Clínica, 37(4), 145-151.

Einsenberg, N., Fabes, R., Guthrie, I., Murphy, B., Maszk, P., Holmgren, R., \& Suh, K. (1996). The relations of regulation and emotionality to problem behavior in elementary school children. Development and Psychopathology, 8(1), 141-162.

Garnefski, N., Kraaij, V., \& Spinhoven, P. (2001). Negative life events, cognitive emotion regulation and emotional problems. Personality and Individual Differences, 30(8), 1311-1327.

George, D., \& Mallery, M. (2010). Using SPSS for Windows step by step: A simple guide and reference. Boston, MA: Allyn \& Bacon.

González, A., Donolo, D., \& Rinaudo, C. (2009). Emociones académicas en universitarios: su relación con las metas de logro. Ansiedad y Estrés, 15(2-3), 263-277.

Gratz, K., \& Roemer, L. (2004). Multidimensional assessment of emotion regulation and dysregulation: Development, factor structure, and initial validation of the difficulties in emotion regulation 
scale. Journal of Psychopathology and Behavioral Assessment, 26(1), 41-54.

Graziano, P., Reavis, R., Keane, S., \& Calkins, S. (2007). The role of emotion regulation in children's early academic success. Journal of School Psychology, 45(1), 3-19.

Gross, J. (1998). The emerging field of emotion regulation: An integrative review. Review of General Psychology, 2(3), 271-299.

Gross, M., Salazar-Jaime, M., Piccolo, N., \& Cupani, M. (2012, octubre). Nuevos estudios de validación del cuestionario de personalidad IPIP-FFM. Trabajo presentado en las Memorias del X Congreso Latinoamericano de Sociedades de Estadística, Córdoba, Argentina.

Gumora, G., \& Arsenio, W. (2002). Emotionality, emotion regulation, and school performance in middle school children. Journal of School Psychology, 40(5), 395-413.

Hervás, G., \& Jódar, R. (2008). Adaptación al castellano de la Escala de Dificultades en la Regulación Emocional. Clínica y Salud, 19(2), 139-156.

Hogan, T. (2004). Pruebas psicológicas. México: Manual Moderno.

John, O., \& Gross, J. (2004). Healthy and unhealthy emotion regulation: Personality processes, individual differences, and life span development. Journal of Personality, 72(6), 1301-1333.

Koole, S. (2009). The psychology of emotion regulation: An integrative review. Cognition and Emotion, 23(1), 4-41.

Ledesma, R., \& Valero-Mora, P. (2007). Determining the number of factors to retain in EFA: An easyto-use computer program for carrying out Parallel Analysis. Practical Assessment Research EG Evaluation, 12(2). Recuperado de http://pareonline.net/ getvn.asp?v $=12 \& n=2$

Lopes, P., Salovey, P., Beers, M., \& Côté, S. (2005). Emotion regulation abilities and the quality of social interaction. Emotion, 5(1), 113-118.

Medrano, A., Moretti, L., Ortiz, A., \& Pereno, G. (2013). Validación del Cuestionario de Regulación Emocional Cognitiva en universitarios de Córdoba, Argentina. Psykhe, 22(1), 83-96.

Medrano, L., \& Moretti, L. (2013). Eficacia de un programa de entrenamiento para disminuir manifes- taciones cognitivas de ansiedad ante los exámenes en ingresantes universitarios. Informes Psicológicos, 13(1), 41-52.

Montero, I., \& León, O. (2007). A guide for naming research studies in psychology. International Journal of Clinical and Health Psychology, 7(3), 847-862.

Newman, A., van Lier, P., Gratz, K., \& Koot, H. (2010). Multidimensional assessment of emotion regulation difficulties in adolescents using the Difficulties in Emotion Regulation Scale. Assessment, 17(1), 138-149.

Niven, K., Totterdell, P., Stride, C., \& Holman, D. (2011). Emotion regulation of others and self (EROS): The development and validation of a new individual difference measure. Current Psychology, 30(1), 53-73.

Pagano, R. (1998). Estadística para las ciencias del comportamiento. México: Thompson.

Pekrun, R., Goetz, T., Titz, W., \& Perry, R. (2002). Academic emotions in students' self-regulated learning and achievement: A program of qualitative and quantitative research. Educational Psychologist, 37(2), 91-105.

Pekrun, R., \& Hofmann, H. (1999). Emotions in learning and achievement: First results of a program of research. En R. Pekrun \& M. Jerusalem (Eds.), Emotion, Motivation und Leistung (pp. 247-267). Göttingen, Germany: Hogrefe.

Pekrun, R., Molfenter, S., Titz, W., \& Perry, R. (2000). Emotion, learning, and achievement in university students: Longitudinal studies. Trabajo presentado en el Annual Meeting of the American Educational Research Association, New Orleans, LA.

Rivas Moya, T. (1999). Comparación de procedimientos para analizar estructuras factoriales en muestras independientes. Revista Electrónica de Metodología Aplicada, 4(1), 19-43.

Rodríguez Ayán, M., \& Ruiz, M. (2008). Atenuación de la asimetría y de la curtosis de las puntuaciones observadas mediante transformaciones de variables: incidencia sobre la estructura factorial. Psicológica, 29(2), 205-227.

Ruganci, R., \& Gencöz, T. (2010). Psychometric properties of a Turkish version of the difficulties in emotion regulation scale. Journal of Clinical Psychology, 66(4), 442-455. 
Salovey, P., Mayer, J., Goldman, S., Turvey, C., \& Palfai, T. (1995). Emotional attention, clarity, and repair: Exploring emotional intelligence using the Trait Meta-Mood Scale. En J. Pennebaker (Ed.), Emotion, disclosure, and health (pp. 125-154). Washington, DC: American Psychological Association.

Sighinolfi, C., Norcini., A., \& Rocco, L. (2010). Difficulties in emotion regulation scale (DERS): The Italian translation and adaptation. Psicoterapia Cognitiva Comportamentale, 16(2), 141-170.

Stewart, S., Zvolensky, M., \& Eifert, G. (2002). The relations of anxiety sensitivity, experiential avoidance, and alexithymic coping to young adults' motivations for drinking. Behavior Modification, 26(2), 274-296.

Tabachnick, B., \& Fidell, L. (2001). Using multivariate statistics (3.a ed.). Boston, MA: Allyn \& Bacon.

Tejeda, M., García, R., González-Forteza, C., \& Palos, P. (2012). Propiedades psicométricas de la escala "Dificultades en la Regulación Emocional" en español (DERS-E) para adolescentes mexicanos. Salud Mental, 35(6), 521-526.
Thompson, B. (2004). Exploratory and confirmatory factor analysis. Washington, DC: American Psychological Association.

Trógolo, M., \& Medrano, L. A. (2012). Personality traits, difficulties in emotion regulation and academic satisfaction in a sample of argentine college students. International Journal of Psychological Research, 5(2), 30-39.

Turner, J., \& Schallert, D. (2001). Expectancy-value relationships of shame reactions and shame resiliency. Journal of Educational Psychology, 93(2), 320-329.

Uriel, E., \& Aldas, J. (2005). Análisis multivariante aplicado. Madrid: Thompson.

Wang, L., Shi, Z., \& Li, H. (2009). Neuroticism, extraversion, emotion regulation, negative affect and positive affect: The mediating roles of reappraisal and suppression. Social Behavior and Personality, 37(2), 193-194.

Ximénez, M., \& García, A. (2005). Comparación de los métodos de estimación de máxima verosimilitud y mínimos cuadrados no ponderados en el análisis factorial confirmatorio mediante simulación Monte Carlo. Psicothema, 17(3), 528-535. 